\title{
Regulatory elements of Xenopus col2a1 drive cartilaginous gene expression in transgenic frogs
}

\author{
RYAN KERNEY*,1, BRIAN K. HALL ${ }^{1}$ and JAMES HANKEN ${ }^{2}$ \\ ${ }^{1}$ Biology Department, Dalhousie University, Halifax, NS, Canada and \\ ${ }^{2}$ Museum of Comparative Zoology, Harvard University, Cambridge, MA, USA
}

\begin{abstract}
This study characterizes regulatory elements of collagen $2 \alpha 1$ (col2a1) in Xenopus that enable transgene expression in cartilage-forming chondrocytes. The reporters described in this study drive strong cartilage-specific gene expression, which will be a valuable tool for further investigations of Xenopus skeletal development. While endogenous col2a1 mRNA is expressed in many embryonic tissues, its expression becomes restricted to tadpole and adult chondrocytes. This chondrocyte-specific expression is recapitulated by col2a1 reporter constructs, which were tested through I-Scel meganuclease-mediated transgenesis. These constructs contain a portion of the Xenopus tropicalis col2a 1 intron, which aligns to a cartilage-specific intronic enhancer that has been well characterized in mammals. Two overlapping regions of the first intron that are 1.5$\mathrm{Kb}$ and 665-bp long, both of which contain this enhancer sequence, drove EGFP expression in both larval and adult chondrocytes when connected to an upstream promoter. However, neither a truncated 155-bp region that also contains the enhancer, nor a separate 347-bp intronic region that lacks it, was able to drive cartilaginous transgene expression. The two cartilage-specific transgenes are heritable in F1 progeny, which exhibit none of the background expression observed in the injected founders. This study is the first to use the I-Scel technique to characterize an enhancer element in Xenopus, and the first to generate chondrocyte-specific gene expression in a nonmammalian vertebrate. The creation of novel cartilage-specific gene expression provides a new tool for further studies of anuran skeletal development.
\end{abstract}

KEY WORDS: col2a1, chondrocyte, meganuclease, Xenopus, sox9

\section{Introduction}

The collagen $2 \alpha 1$ gene (col2a1) encodes type-Il collagen, the primary collagen protein of cartilage. The gene product, a pro $\alpha 1$ (II) polypeptide, forms helical homotrimers through its collagenous domain in the mature type-Il collagen protein (reviewed in Boot-Handford and Tuckwell, 2003). The high copy number of individual polypeptides required for the collagen-rich extracellular matrix of cartilage coincides with high copy number of precursor mRNA within individual chondrocytes (Kosher et al., 1986a; Kosher et al., 1986b). Col2a1 mRNA is also expressed in the notochord, somites, lateral plate mesoderm, and ventral neural tube in Xenopus laevis (Su et al., 1991; Kerney et al., 2007) and mouse embryos (Thorogood et al., 1986; Wood et al., 1991). Expression of col2a1 in $X$. laevis becomes restricted to the cartilaginous skeleton of the tadpole and notochordal sheath during skeletogenesis (Su et al., 1991). An alternatively spliced isoform, which lacks the second exon, also occurs during carti- lage-forming stages in Xenopus (Su et al., 1991) and mouse (Sandell et al., 1991).

Investigations into the regulation of col2a1 transcription in mammals have concentrated on its mRNA expression in chondrocytes. These studies reveal an enhancer region in the first intron that is necessary for chondrocyte-specific expression of col2a1 mRNA. Successive truncations have reduced this intronic en-

\footnotetext{
Abbreviations used in this paper: AC, alary cartilage; $\mathrm{AR}$, articular chondrocytes; $\mathrm{BA}$, branchial arches; $\mathrm{CB}$, ceratobranchial cartilages; $\mathrm{CH}$, ceratohyal cartilage; col2a1, collagen $2 \alpha 1$ gene; EGFP, enhanced green fluorescent protein; EY, eye; FP, floor plate of the neural tube; GFP, green fluorescent protein; HC, hypertrophic chondrocytes; HU, humerus; HMG, high mobility group; IH, interhyoideus muscle; MC, Meckel's cartilage; NC, nasal capsule; NF, Nieuwkoop Faber Stage; NO, notochord; NS, nasal septum; OV, otic vesicle; PA, planum antorbitale; PC, proliferating chondrocytes; PT, planum terminale; RU, radio-ulna; SO, somites; SPC, superior prenasal cartilage; UTR, untranscribed region.
}

*Address correspondence to: Ryan Kerney. 1355 Oxford St., Biology Department, Dalhousie University, Halifax, NS, Canada Fax: +1-902-494-3736. e-mail: ryankerney@gmail.com - web: http://web.mac.com/ryankerney/iWeb/Site/Welcome.html 
hancer sequence from over 3-Kb to 800-bp (Horton et al., 1987), 182-bp (Zhou et al., 1995), 48-bp (Zhou et al., 1998) and 18-bp (Lefebvre et al., 1996) regions, each of which is capable of driving chondrocyte expression when connected to a col2a1 promoter. The mouse minimal enhancer contains the HMG recognition sequence, CATTCAT, which binds to the HMG-box containing transcription factor Sox9 (Lefebvre et al., 1997). However, multiple copies of the truncated $48 \mathrm{bp}$ and $18 \mathrm{bp}$ enhancer regions are required for detectable expression of reporter genes, indicating the presence of additional regulatory information outside the Sox9-binding region.

The tissue-specificity and high level of expression generated by the col2a1 promoter/enhancer have provided a valuable tool for studies of gene function within the chondrocyte lineages of mice. Variations of this reporter construct have been used for over-expression of dominant-negative and full-length genes within mouse chondrocyte lineages (Nakata et al., 1993; Tsumaki et al., 1999; Takeda et al., 2001; Ueta et al., 2001; Stricker et al., 2002). The mouse reporter construct also has been fused to both doxycycline and tamoxifen-inducible enhancers, allowing temporal control of chondrocyte-specific gene expression (Grover and Roughley, 2006; Nakamura et al., 2006; Chen et al., 2007).

To date, the use of a cartilage-specific reporter has been limited to studies in mammals. No other vertebrate taxon has a similar tool for driving transgene expression in chondrocytes. The development of a col2a1 reporter in alternative species (and classes) would allow investigations of both comparative gene
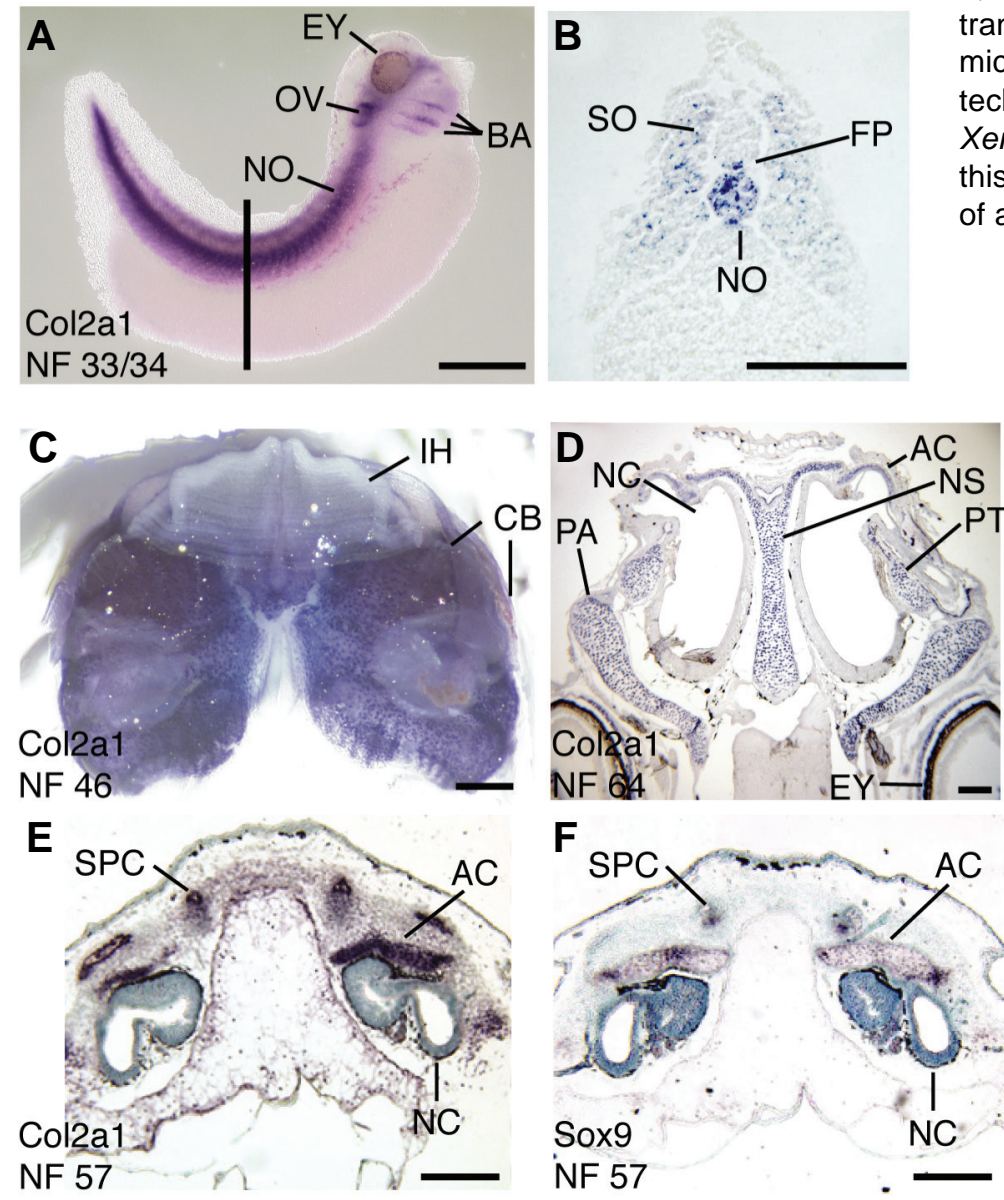

regulation and comparative skeletal development. Metamorphosing anurans are ideal taxa for this type of investigation given their unique skeletal development, which is associated with a biphasic life history.

Metamorphic development of the anuran skeleton differs from that of amniotes in having two discrete periods of skeletogenesis. The first, embryonic phase of skeleton formation yields the strictly cartilaginous tadpole skeleton. The second, metamorphic phase of skeleton formation results in both new cartilage and bone formation in the post-metamorphic froglet (reviewed in Hanken, 1992). Previous investigations have concentrated on the cellular origins of the tadpole skull (Stone, 1929; Sadaghiani and Thièbaud, 1987; Olsson and Hanken, 1996) and the differentiation of tadpole chondrocytes (Spokony et al., 2002; Baltzinger et al., 2005; Kerney et al., 2007). Few studies, however, have investigated the cellular origins and differentiation of chondrocytes during the second-metamorphic-phase of anuran skull development (e.g., Gross and Hanken, 2008). This lack of research is largely attributable to the long period required for tadpoles to complete metamorphosis, and a lack of long-term genetic tools needed to manipulate and analyze metamorphic processes.

This study investigates the regulation of col2a1 in Xenopus using I-Scel meganuclease-mediated transgenics and provides a tool for future investigations of anuran skeletal development. This transgenic technique was originally used in a teleost, the Japanese medaka (Oryzias latipes; Thermes et al., 2002), and later adapted for the creation of transgenic Xenopus (Ogino et al., 2006 $a, b ;$ Pan et al., 2006). The technique allows for the efficient transfection of embryos through simple enzyme digestion and microinjection. This study is the first to employ this transgenic technique for the characterization of an enhancer element in Xenopus. The cartilage-specific reporter construct described in this study will facilitate further investigations into the genetic basis of anuran skeletal development and metamorphosis.
Fig. 1. In situ hybridizations during embryonic (A,B), larval (C), metamorphic (E,F) and post-metamorphic stages (D). (A,B) Whole-mount col2a1 in situ hybridization of a NieuwkoopFaber (NF) stage-33/34 embryo. (A) Lateral view, anterior is to the right. There is strong expression of col2a 1 in the notochord (NO), otic vesicle (OV) and branchial arches (BA). Black line points to level of section depicted in (B). (B) Histological cross section through the trunk. Note diffuse staining in the somites (SO) and floor plate of the neural tube (FP). (C) Whole-mount col2a1 in situ hybridization; stage-46 tadpole head. Ventral view, anterior is to the top. There is strong expression in early larval cartilages, including the ceratobranchials (CB), but not in the interhyoideus muscle (IH). (D) Sectioned in situ hybridization of a stage-64 froglet nasal capsule. Coronal section, anterior is to the top. Col2a1 is expressed again in the adult cranial cartilages, including the post-metamorphic nasal septum (NS), planum terminale (PT) planum antorbitale (PA), and alary cartilage (AC). (E,F) Midmetamorphic, stage-57 tadpole. Coronal histological sections of the nasal capsule, stained byin situ hybridizations with col2a1 and sox9 probes. (E) Col2a1 expression increases in the alary (AC) and superior prenasal (SPC) cartilages as they form during metamorphosis. (F) Up-regulation of col2a1 corresponds with heightened expression of sox9 in these cartilages during metamorphic chondrogenesis. Additional abbreviations: NC, nasal capsule; EY, eye. Scale bar, $0.5 \mathrm{~mm}$ 


\section{Results}

\section{In situ hybridizations}

After an initial distribution in several embryonic tissues, expression of col2a1 mRNA becomes restricted to the cartilaginous skeleton of the tadpole and later of the adult frog. In stage 33/34 embryos, col2a1 is expressed in the floor plate of the neural tube, the notochord, branchial arches, somites, and otic vesicles (Fig. $1 \mathrm{~A}, \mathrm{~B})$. By stage 46 , col2a1 is strongly and exclusively expressed in larval chondrocytes (e.g., ceratobranchial cartilages; Fig. 1C). During metamorphosis, new cartilages contribute to the adult nasal capsule and sound-conducting plectral apparatus (Trueb and Hanken, 1992; Gross and Hanken, 2008). Metamorphic formation of alary and superior prenasal cartilages in the nasal capsule corresponds with heightened col2a1 mRNA expression (Fig. 1E) along with coexpression of the col2a1 regulator sox9 (Fig. 1F). By stage 64, col2a 1 is strongly expressed in post-metamorphic cartilages of the froglet, including the nasal capsule (Fig. 1D).

\section{Promoter characterization}

Previous research on the chondrocyte-specific expression of Col2a1 in mammals indicated a synergism between the 5' promoter and intronic enhancer (Savagner et al., 1990; 1995; Leung et al., 1998; Seki et al., 2003). Therefore, 491 bp of upstream sequence from Xenopus tropicalis was used as a promoter in each transgene construct characterized in this study (Fig. 2A). This sequence includes 366 bp of regulatory DNA and 125 bp of 5 -untranslated region (UTR). A LAGAN/Vista analysis revealed no significant sequence similarity between the mouse and $X$. tropicalis promoter regions (data not shown). However, a sequence 13 bp upstream of the transcription start site (TATAACT) in $X$. tropicalis is identical to the TATAbox sequence of the mouse Col2a1 promoter (Fig. 2A), but not the human COL2A1 TATA-box (TATAACGG) (Osaki et al., 2003). While both the human and mouse Col2a1 promoters share a TFIIB recognition sequence (GGGMGCA) upstream of the TATA-box (Osaki et al., 2003), this sequence is not found in the $X$. tropicalis promoter. The $X$. tropicalis col2a 1 promoter region also contains a putative binding site for the delta EF1 transcription factor, based on a TFSearch analysis $(95 \%$ minimal threshold score with vertebrate matrices; http://www.cbrc.jp/research/ $\mathrm{db} /$ TFSEARCH.html; Heinemeyer et al., 1998), which is not found in the mouse or human promoter (Fig. 2A).

\section{Intron characterization}

The LAGAN/Vista alignment of the first col2a1 intron from mouse and Xenopus tropicalis reveals a 144-bp sequence that aligns with $74.3 \%$ sequence identity (Fig. 2B, C). This region overlaps only the first 69 base pairs of the 182-bp mouse enhancer (Zhou et al., 1995). However, the truncated 48-bp and 18-bp (Lefebvre et al., 1996) enhancer sequences are con-

B tained within the aligned $X$. tropicalis sequence. The 48-bp mouse enhancer contains two inverted repeats that are conserved with the rat and human Col2a1 intronic sequences (Lefebvre et al., 1996). Only the outer repeat (TGAAT) is conserved with $X$. tropicalis (Fig. 2C). Its inverted repeat (ATTCA) appears 30 base pairs downstream and is within the seven-base Sox9 binding region (CATTCAT; Lefebvre et al., 1997), which is strictly conserved between mouse and $X$. tropicalis. Two additional putative
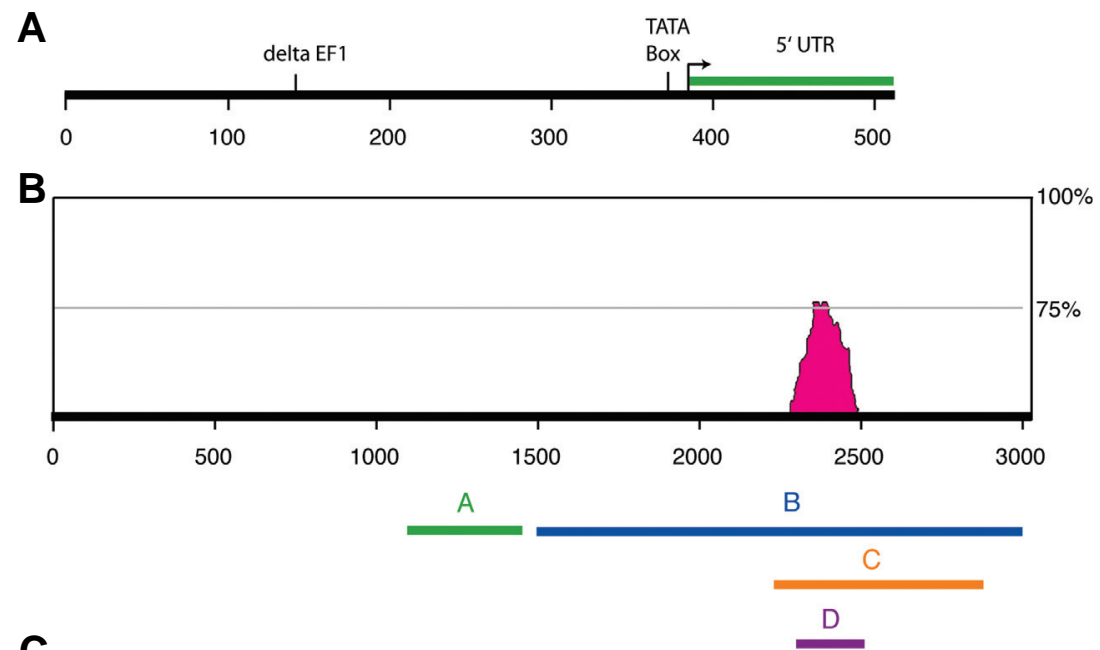

Vista Conserved Region. Start; 2319, Length; 144 bp, Identity; 74.3\%

Xenopus TTGTGATGGTTATTTGCACTGGGAGGATTGGAGCAATGGTTTCCAGATGGAGTGAAATG Mouse TTGTGA-GACTTGTTTGCGTTGGG-GGATTGGCAGCGATGGCTTCCAGATGG-GCTGAAACC

Xenopus CTGCCCATGTTTATTTAAACTGGCCC CTGACAGT GAAATg TGAAT TGA ACCTCTG CTGCCCGTATTTATTTAAACTGGTTC CTCGTGGA GAGCTG IGAAT CGGG CTCTGTA

Xenopus TGTATTTTACAAAAAGGCCC ATTCA TTAAG $\begin{array}{lllll}\text { Mouse } & \text { TGCGCTTGA-GAAAAGCCCC } & \text { ATTCA } & \text { TGAGA } \\ \text { ATTCA }\end{array}$

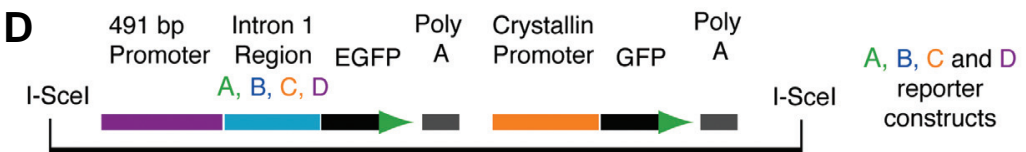

Fig. 2. Diagrams of the regulatory elements used in this study. (A) The 491-bp, 5'promoter region used in the reporter includes $366 \mathrm{bp}$ of untranscribed DNA and $125 \mathrm{bp}$ of the 5' UTR. A TFSearch analysis (Heinemeyer et al., 1998) reveals a putative transcription-factor binding site for delta EF1 (-243 bp from the transcription start site). A TATA-box sequence occurs -13 bp from the transcription start site. (B) Lagan/Vista plot of alignments between $\mathrm{X}$. tropicalis and mouse col2a1 first introns. Base pair positions of aligned sequence along the $X$-axis; the $Y$-axis depicts percentage sequence identity. The line graph shows a 144-bp alignment region that begins at $\mathrm{X}$. tropicalis position $2319 \mathrm{bp}$. Intron fragments, represented by colored bars beneath the alignment, were tested in reporter constructs. (C) Nucleotide sequences of aligned region in (B) with conserved nucleotides shown in red; gaps and substitutions are black. The two yellow boxes correspond to two putative HMG binding sites of weak affinity described from the mouse sequence (Zhou et al., 1998). The red underline corresponds to the nucleotide region that is essential for binding Sox9 protein to the mouse enhancer (Lefebvre et al., 1997). The purple boxes correspond to a conserved inverted repeat (Lefebvre et al., 1996), which is separated by 30 bp and has a downstream portion inside the Sox9 binding region. (D) Schematic depiction of the reporter constructs used in this study. The col2a1 $5^{\prime}$ promoter region was connected to the $(A, B, C$, and $D)$ intronic sequences to drive the expression of EGFP. A separate gamma-crystallin promoter was used to drive lens-specific expression of GFP as a positive control for transfection of injected embryos. Two 18-bp I-Scel recognition sites flank each reporter construct. 

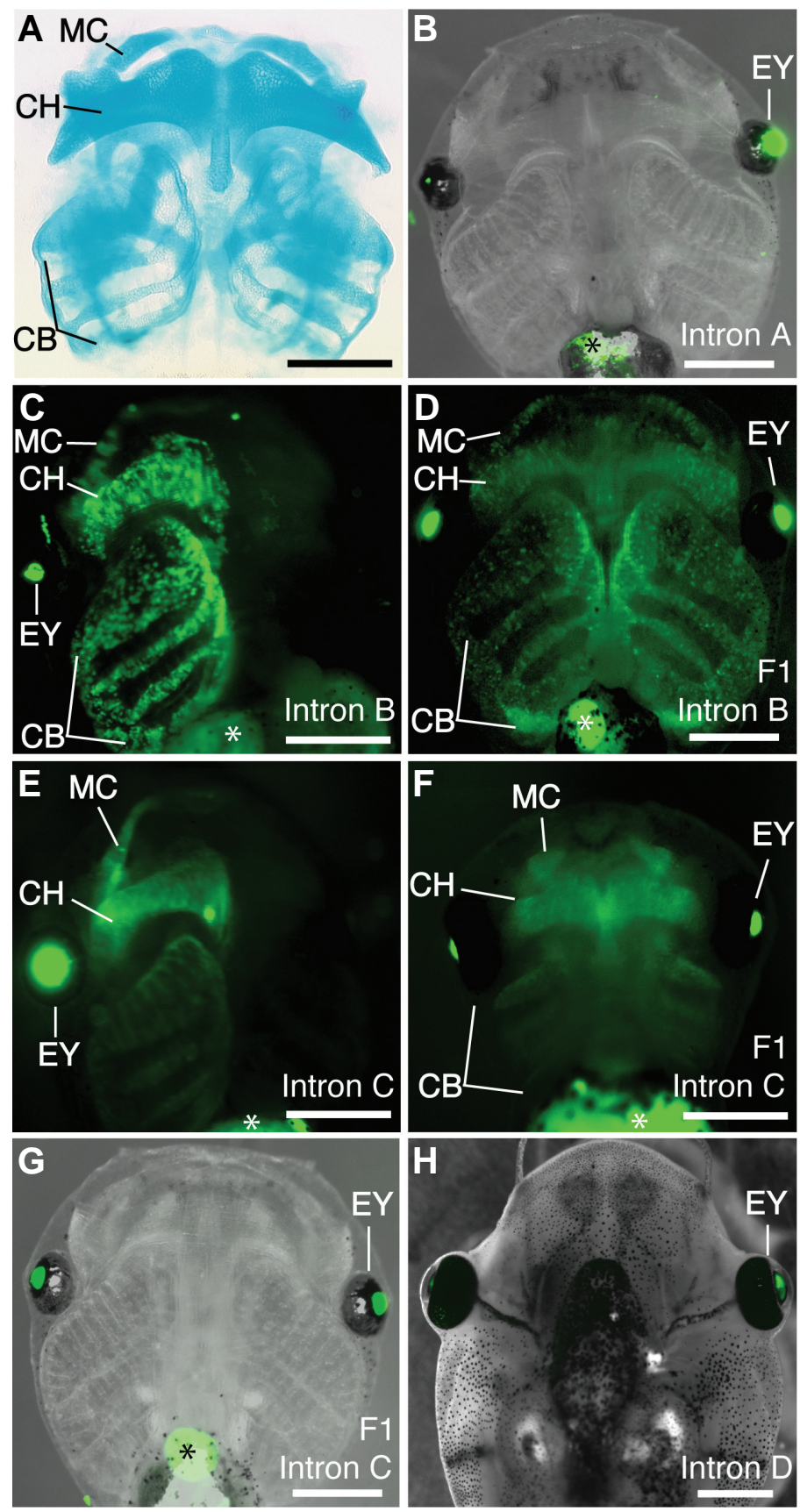

HMG binding sites (Zhou et al., 1998) are not conserved between mouse and $X$. tropicalis intronic sequences (Fig. 2C).

\section{I-Scel meganuclease-mediated transgenesis}

A region of the col2a1 promoter and $5^{\prime}$ UTR was connected to three separate regions of the first col2a1 intron and linked to egfp. Each construct was injected into zygotes of Xenopus laevis along with I-Scel meganuclease. The resulting embryos were screened for separate lens-specific GFP expression under the control of the gamma-crystallin promoter, which was used as a positive control for transgene uptake (Rankin et al., 2009). Injection of each construct generates EGFP expression in a subset of epidermal cells early in development in most injected animals (Table 1). This
Fig. 3. Distribution of EGFP in transgenic Xenopus laevis tadpoles. (A-G) Ventral views, anterior to the top. (H) Dorsal view, anterior to the top. (A) Cleared-and-stained tadpole showing Meckel's (MC), ceratohyal (CH) and ceratobranchial (CB) cartilages stained with Alcian blue (stage 42). All other images show EGFP/GFP expression in living tadpoles. (B) Transient transgenic tadpole with a reporter carrying the intronic region A (stage 48). EGFP is not seen in the cartilaginous skeleton of any tadpole carrying reporter construct $A$. GFP expression is apparent in the lens of the left eye (EY), under the control of the gamma-crystallin promoter. (C) Transient "half-transgenic" tadpole with a reporter carrying the intronic region $B$ (stage 42). Unilateral transgene expression is apparent in the same cartilages labeled in (A), along with GFP expression in the eye. (D) An F1 transgenic tadpole (stage 48), bred from a half-transgenic mother carrying construct $B$. (E) Transient half-transgenic tadpole carrying a reporter with the $C$ construct (stage 42) also exhibits unilateral cartilaginous expression, although expression is weaker than that seen in tadpoles carrying the B construct. (F) An F1 transgenic tadpole (stage 40), bred from a half-transgenic mother carrying construct $C$. Early expression of EGFP is strongest as the cartilages are forming. (G) Stage 48 of the same tadpole in (F). None of the tadpole cartilages continues to express EGFP under construct $C$ during the later larval stages. However, cartilaginous expression of EGFP increases again during the metamorphic formation of adult cartilages (Fig. 4). (H) Merged bright-field and fluorescent image of a transgenic tadpole carrying a reporter with the smaller intronic region D (stage 48). This region does not drive cartilaginous EGFP expression during any stage in any injected tadpole. Auto-fluorescence is visible in the yolk of stage 40-42 individuals (asterisks in $C, E, F$ ) and in the gallbladder of stage-48 tadpoles (asterisks in B,D,G). Scale bar, $1 \mathrm{~mm}$.

shared expression may be due to expression driven by the $5^{\prime}$ promoter region itself, or it may be an artifact of the egfp injection (Pan et al., 2006). No construct drives detectable EGFP expression similar to the col2a1 mRNA expression in the notochord, otic vesicle, somites, floor plate of the neural tube, or branchial arches during early development (Fig. 1A, B).

The 347-bp intronic region ' $A$ ' does not overlap with the aligned region from our Vista analysis (Fig 2B). This sequence was used as a negative control and compared to the expression generated by intronic sequences that contain the aligned enhancer. $A$ reporter construct containing intronic sequence $A$ does not drive expression outside the lens and early epidermal cells (Fig. 3B, Table 1). Epidermal expression dissipates in later-stage tadpoles. No other pattern of EGFP is found in any additional larval tissue.

The reporter construct that contains the 1,517-bp region 'B' of the first intron (Fig. 2B) drives EGFP expression in the cartilaginous skeleton (Fig. 3C-D, 4A-F) in 24 of 70 transgenic animals (34.3\%; Table 1). Twelve of these have bilateral mosaic expres-

TABLE 1

\section{EXPRESSION OF EGFP IN TADPOLES INJECTED WITH FOUR REPORTER CONSTRUCTS}

\begin{tabular}{cccc} 
Intronic Region & Length & Total Transgenic & \% Chondrocyte Expression \\
\hline A & $347 \mathrm{bp}$ & 22 & 0 \\
B & $1,517 \mathrm{bp}$ & 70 & 34 \\
C & $665 \mathrm{bp}$ & 78 & 26 \\
D & $153 \mathrm{bp}$ & 22 & 0 \\
\hline
\end{tabular}

"Total transgenic" refers to the number of injected tadpoles with GFP expressed in the lens under the gamma-crystallin promoter (positive control). Chondrocyte expression in transgenic tadpoles (with lens-specific GFP expression) was scored between stages 42 and 47 for each intronic region assayed. 

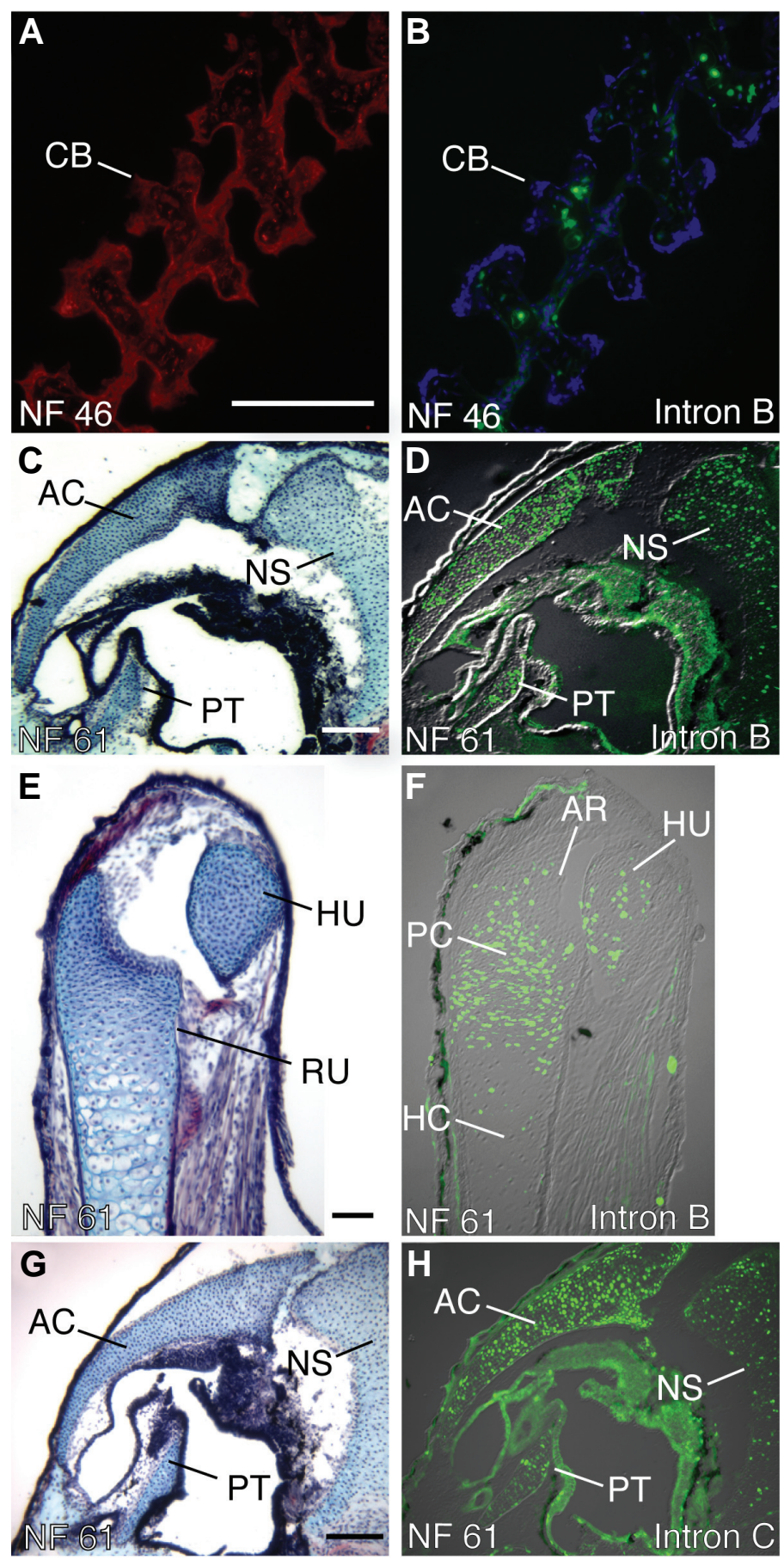

sion within cartilage (data not shown), while the other twelve show nearly complete expression within cartilage that is distributed on only one side of the tadpole (Fig. 3C, 4B). The stronger unilateral expression indicates continued integration of the transgene after the first cell division. Cartilaginous EGFP expression co-localizes with collagen-Il antibody reactivity in the ceratobranchial cartilages (Fig. 4A-B). Expression of EGFP driven by this construct is strong during early cartilage-forming stages (40-42) but decreases later (stage 48-metamorphosis). This may be due to an increase in production of the extracellular matrix and spreading of the chondrocyte lacunae, or to weaker activation of the col2a1 regulatory
Fig. 4. Chondrocyte expression of reporter constructs in histological sections. (A,B) Tadpole injected with a reporter construct that contains intronic region $B$. Coronal sections through a single larval ceratobranchial cartilage stained with anti-collagen II antibody (red in A) or anti-GFP antibody (green in B) with DAPI nuclear counter stain (b/ue). (C,E,G) Trichrome and (D, F, H) merged bright-field and fluorescent images of anti-GFP antibody stained sections. (C,D) Coronal sections of the nasal capsule of a post-metamorphic froglet that carries the reporter construct with intronic region $B$. (D) EGFP is expressed in the alary cartilage (AC), nasal septum (NS) and planum terminale (PT). (E,F) Longitudinal sections through the leg of a froglet that carries a reporter construct with intronic region $B$. Cartilaginous expression is seen in the humerus (HU) and proliferating chondrocytes (PC) of the radio-ulna (RU). EGFP is not expressed in the hypertrophic $(H C)$ or articular (AR) chondrocytes of the radio-ulna. $\mathbf{( G , H ) ~ S e r i a l ~ c o r o n a l ~ s e c t i o n s ~ t h r o u g h ~ t h e ~ e t h m o i d a l ~ r e g i o n ~ o f ~}$ a froglet that carries the $C .1$ reporter. Cartilaginous expression is seen in the same post-metamorphic cartilages shown in D. Scale bar, $0.25 \mathrm{~mm}$.

sequences in later stages. Cartilaginous EGFP expression increases in post-metamorphic cartilages of the nasal capsule (Fig. 4 C-D), which also express col2a1 mRNA (Fig. 1D-E). Proliferative chondrocytes of the radio-ulna and humerus also express EGFP under this reporter (Fig. 4 E-F). EGFP is not expressed, however, in either articular or hypertrophic chondrocytes of the radio-ulna (Fig. 4F). Col2a1 mRNA expression diminishes in hypertrophic chondrocytes of mice and chickens, where type II collagen is largely replaced by type $X$ collagen before endochondral ossification (reviewed in Goldring et al., 2006). Seven tadpoles express EGFP in a small number of axial myocytes; this gradually diminishes in later tadpole stages.

A half-transgenic female carrying the $B$ construct was bred to a wild-type male. Bilateral cartilaginous EGFP expression is observed in 24 of 110 progeny. These 24 individuals consistently express EGFP in the cartilaginous skeleton with no variation among individuals, which indicates a single integration of the transgene (Marsh-Armstrong et al., 1999). Early chondrocyte expression is strong (stages 40-42). Later EGFP expression (stage 48) is reticulate within individual cartilages (Fig. 3D).

The reporter construct with the 665-bp intronic region ' $C$ ' also drives EGFP expression in the cartilaginous skeleton, although its expression is less intense than that generated by intronic region B (Figs. 3E-G, 4G-H). EGFP-expressing chondrocytes occur in 20 of 78 tadpoles transfected with this reporter (25.6\%; Table 1). Nine of these have stronger unilateral expression (Fig. 3E), while eleven exhibit weaker sporadic expression that is distributed bilaterally in the early cartilaginous skeleton. Early cartilaginous expression of EGFP dissipates by stage 48 and is undetectable in later-stage chondrocytes (Fig. 3G). As with construct B, however, EGFP is strongly expressed in metamorphic cartilages of the nasal capsule in froglets that carry construct $C$ (Fig. $4 \mathrm{H}$ ). Myocyte expression also occurs in ten of the injected tadpoles, although it dissipates in later stages.

A half-transgenic female carrying the $C$ construct, again with strong unilateral EGFP expression, was bred to a wild-type male. The resulting progeny have bilateral cartilaginous expression of EGFP by stage 40 (Fig. 3F), which dissipates entirely by stage 48 (Fig. 3G). The transgene was inherited in approximately half (18 of 30 ) of the $F 1$ progeny. The $F 1$ generation of construct $C$ exhibits more variation in the distribution of EGFP and has twice the proportion of transgenic individuals compared to the F1 genera- 
tion of construct $B$, which indicates multiple transgene insertions in the maternal founder. Despite this increased number of insertions, cartilaginous expression of EGFP generated by construct $C$ is weaker than that generated by construct $B$ in stage-matched, F1 progeny (Fig. 3D, G).

The reporter construct that carries the 155-bp intronic region ' $D$ ' does not drive expression in any tissue outside the early epidermis (Fig. $3 \mathrm{H}$ ). Although this construct contains the entire aligned intronic enhancer region (Fig. 2B), it fails to drive EGFP expression in myocytes or chondrocytes of 22 transgenic tadpoles, all of which express GFP in the lens under the control of the gamma-crystallin promoter (Table 1).

\section{Discussion}

This study describes the expression of col2a1 mRNA in larval, metamorphic and post-metamorphic Xenopus laevis and characterizes reporter constructs that use a transcriptional enhancer element from $X$. tropicalis' col2a 1 in $X$. laevis tadpoles and adults. This enhancer element is located in the 3' region of the first intron and aligns closely with a well-characterized chondrocyte-specific mouse enhancer. The Xenopus enhancer, combined with a fragment of the 5' promoter, drives cartilaginous EGFP expression in I-Scel meganuclease-mediated transgenic tadpoles and adults. These transgenes are heritable through the germ line and are expressed both in larval cartilages that form during embryogenesis and in adult cartilages, which form de novo during metamorphosis and are not present in the larval stage.

Whereas several studies examine the early distribution of col2a1 mRNA in Xenopus laevis (Su et al., 1991; Bieker and Yazdani-Buicky, 1992; Larrain et al., 2000; Kerney et al., 2007), this study is the first to investigate its expression through metamorphosis (Fig. 1). An initially widespread embryonic expression of col2a1 becomes restricted to the cartilaginous skeleton of the larva and continues to be strongly expressed in chondrocytes during subsequent stages. Formation of adult cartilages in the nasal capsule during metamorphosis coincides with an increase in mRNA expression of both col2a1 and the transcription factor sox9. This transcriptional regulator is essential for chondrocyte differentiation (Akiyama et al., 2002) and early col2a1 expression (Lefebvre et al., 1997) in mice. Expression of sox9 during metamorphic chondrogenesis may either coincide with the late differentiation of mesenchymal precursors into functioning chondrocytes or reflect an increase of type II collagen production by existing larval chondrocytes to create new cartilage. Post-metamorphic cartilages of the nasal capsule continue to express col2a1 mRNA after they are fully formed.

Several studies in mammalian cell culture characterize other positive and negative regulatory elements in the Col2a 1 promoter region (Savagner et al., 1990, 1995; Seki et al., 2003; Osaki et al., 2003). An Sp1 binding region (Savagner et al., 1995) and two Ebox silencer regions in the rat promoter (CIIS1 and CIIS2; Savagner et al., 1990) are not found in the $3.6 \mathrm{~Kb}$ upstream sequence of the $X$. tropicalis promoter (data not shown). However, the presence of a putative delta EF1 binding site in the Xenopus tropicalis promoter (Fig. 2A) indicates a possible site of transcriptional regulation. Delta EF1 is a zinc finger homeobox-1 transcription factor $(z f h \times 1)$. Null mutant mice that lack functional Delta EF1 have fused limb cartilages and fusions of the carpal and tarsal cartilages in neonates (Takagi et al., 1998). Delta ef1 mRNA is expressed in paraxial mesoderm, migratory cranial neural crest, retina and neural tube of Xenopus laevis embryos (van Grusven et al., 2006). Delta EF1 acts as both a repressor of E2-box mediated transcription in mice (Sekido et al., 1994) and Smadmediated BMP signaling in Xenopus (van Grusven et al., 2006). However, studies of the mouse Col2a1 promoter have not identified Delta EF1 as a potential transcriptional silencer. Further research in the binding of Delta EF1 to the Xenopus promoter is needed to validate its role in col2a1 regulation and anuran skeletogenesis.

Lagan/Vista alignment of the first intron from col2a1 in mouse and Xenopus tropicalis reveals a 144-bp region with $74.3 \%$ sequence identity. This sequence contains the seven-base Sox 9 binding region from the mouse intron (CATTCAT; Lefebvre et al., 1997), which is strictly conserved with the $X$. tropicalis sequence. Part of this recognition sequence exists as an upstream inverted repeat, which also is found in the mouse enhancer (Lefebvre et al., 1996). Pairs of inverted Sox-binding sites have been characterized in the enhancer of a precursor gene for type XI collagen, col11a2. These are essential for chondrocyte-specific transcriptional activity in col11a2 as well (Bridgewater et al., 2003). However, additional sequences outside the repeated binding domains are required for the col11a2 enhancer to drive transgene expression in chondrocytes. Other regulatory information may also exist outside the conserved col2a1 enhancer region. Together, Sox6 and L-Sox5 cooperate with Sox9 to activate col2a1 expression in chondrocytes (Bell et al., 1997). Their binding sites in the col2a1 regulatory regions have not been identified in either mammals (Lefebvre and Smits, 2005) or X. tropicalis.

While the one established Sox9 binding site in the col2a1 enhancer is conserved between mouse and Xenopus, two additional putative HMG binding sites (Zhou et al., 1998) are not (Fig. 2C). The Sox9 protein does not bind to either of these putative sites in the mouse intron (Lefebvre et al., 1997). Absence of these sites from the Xenopus sequence further suggests that these sites are not part of the Sox9 regulation of col2a1.

Regulatory ability of the intronic enhancer from Xenopus tropicalis was tested in $X$. laevis through I-Scel meganucleasemediated transgenesis. A large $1.5-\mathrm{Kb}$ region of the intron drove cartilaginous expression in both tadpole and adult cartilages when connected to an upstream promoter (Fig. 2B, region B). While a smaller 665-bp region also drove cartilage-specific expression, its expression was weaker than the larger intronic fragment $B$ (Fig. 2B, region C). A truncated 155-bp region containing the entire aligned sequence from the LAGA/Vista analysis failed to drive any expression (Fig. 2B, region $\mathrm{D}$ ). These results are similar to previous studies of the mouse Col2a1 intronic enhancer, in which multiple copies of smaller enhancer regions are required to drive cartilaginous gene expression (Lefebvre et al., 1996; Zhou et al., 1998). Multiple copies of region D were not tested in this study.

The negative-control construct $A$, which contains the $5^{\prime}$-promoter (491 bp) but not the conserved intronic enhancer, failed to show any EGFP expression in chondrocytes. Intronic region $A$ (347 bp) is shorter than $B(1.5 \mathrm{~Kb})$ and $C$ (665 bp) regions that do drive cartilage-specific expression. The small size of $A$ may account for its inability to drive cartilage-specific expression. The decreasing sizes of regions $\mathrm{B}, \mathrm{C}$ and $\mathrm{D}$ correspond with $\mathrm{a}$ 
decrease in their effectiveness in driving cartilage-specific expression (Table 1). This trend has been observed in studies of mouse Col2a1 regulation, where smaller enhancer-containing fragments require multiple copies to activate transcription (Zhou et al., 1998; Lefebvre et al., 1996). However no study of a mammalian col2a1 first intron has revealed regulatory information outside the Sox9-binding enhancer region. The rat col2a15'promoter (310 bp) is capable of driving faint expression in chondrocytes without the intronic enhancer (Horton et al., 1987). However, neither $6.1 \mathrm{~Kb}$ of the human promoter (Leung et al., 1998) nor 309 bp of the mouse promoter (Zhou et al., 1995) drives chondrocyte expression without the intronic enhancer in transgenic mice. Xenopus, like human and mouse, appears to require intronic enhancer sequence for chondrocyte-specific expression.

The mouse enhancer also can drive chondrocyte-specific expression when connected to a minimal human $\beta$-globin promoter (Zhou et al., 1995). Surprisingly, the Col2a1 enhancer (4 copies of $264 \mathrm{bp}$ ) from the extinct "marsupial wolf" Thylacinus cynocephalus can drive chondrocyte-specific transgene expression in transgenic mice when connected to the $\beta$-globin promoter (Pask et al., 2008). However, a similar $\beta$-globin promoter construct, with 308 bp of the human COL2A1 intronic enhancer, does not drive chondrocyte-specific expression in mice (Leung et al., 1998).

Expression of EGFP in $X$. laevis tissues under the control of $X$. tropicalis regulatory information further supports the previously suggested compatibility of the two species (Khokha et al., 2002). $Z y$ gotes of $X$. laevis are larger and easier to microinject than those of $X$. tropicalis. However, the longer generation time in $X$. laevis makes germ line transmission of transgene constructs difficult to test within a reasonable time frame. Founder frogs bred in this study required 18 months to reach sexual maturity in the laboratory. Compatibility in regulatory sequence function between these species also suggests the possibility that the col2a1 reporters described here will drive transgene expression in cartilages of other amphibians. The I-Scel meganuclease method of transgene integration works in the Mexican axolotl, Ambystoma mexicanum (Sobkow et al., 2006), and similar microinjection techniques have been developed for the Oriental fire-bellied toad, Bombina orientalis (Olsson and Hanken, 1996; Olsson et al., 2001), which suggests the feasibility of testing the col2a1 reporter in a wide number of alternative amphibian models.

The meganuclease technique does yield some mosaic expression of EGFP among individual chondrocytes for each reporter tested. This mosaic expression is dramatically reduced in "halftransgenic" tadpoles, which strongly express EGFP unilaterally on either the left or right side. EGFP expression is consistently more intense in the cartilages of half-transgenic tadpoles than in "full-transgenic" individuals, which express cartilaginous EGFP bilaterally (Fig. 3). Half-transgenic individuals result from incorporation of the transgene after the first cellular division. The transgene is heritable provided the germ line cells carry the inserted construct (Pan et al., 2006).

Background expression from the reporter construct was observed in epidermal cells and larval myocytes. It is reduced in older tadpoles and post-metamorphic froglets, and absent in F1 progeny. This pattern indicates that the background is a result of injected egfp DNA, and not due to integrated transgene expression.

\section{Conclusions}

Cartilage-specific expression generated by these reporter constructs provides a useful tool for future investigations of anuran skeletal development. These constructs can be used to drive dominant-negative protein expression or to over-express various genes in the cartilaginous skeleton. Whereas several studies investigate the skeletal morphology of Xenopus laevis during development (Bernasconi, 1951; Sedra and Michael, 1957; Sokol, 1977; Trueb and Hanken, 1992), few examine the underlying transcriptional regulators involved in skeletogenesis (Pasqualetti et al., 2000; Spokony et al., 2002; Moriishi et al., 2005; Kerney et al., 2007). Despite ongoing use of Xenopus as a model system of organogenesis (Thomsen, 2006) and the quickly growing field of skeletal developmental biology (Karsenty, 2003; Zelzer and Olsen, 2003; Hall, 2005; Goldring et al., 2006) few studies research skeletal development in Xenopus. This lack of attention is due largely to the limited array of techniques available for investigations of later development in Xenopus (Blitz et al., 2005). Functional experiments through the injection of morpholino oligonucleotides, antisense RNA, or RNA interference are limited to early stages (Nutt et al., 2001; Khokha et al., 2002). Due to these limitations, there are no current investigations into the function of skeletal regulatory genes during anuran metamorphosis. The ability to drive chondrocyte-specific gene expression in Xenopus will provide a valuable tool for future investigations of gene function and cell lineage in anuran skeletal development.

\section{Materials and Methods}

All animal protocols were approved by the Harvard University Faculty of Arts and Sciences Standing Committee on the use of Animals in Research and Teaching (protocol 99-09) and Dalhousie University's Committee on Laboratory Animals (protocol 07-126).

\section{In situ Hybridizations}

Antisense mRNA probes were prepared from pCS2-AS-collagen II (Larrain et al., 2000) and pGEMT:Sox9 (Spokony et al., 2002). Wholemount in situ hybridizations were performed on albino embryos and hatchlings following the protocol of Sive et al. (2000). Embryos were fixed in MEMFA and stored in $70 \%$ methanol at $-20^{\circ} \mathrm{C}$ prior to initial use. In situ hybridizations on sectioned tissues followed the protocol of Zelzer et al. (2001). Sectioned tissues were fixed in 4\% paraformaldehyde in phosphate-buffered saline $(\mathrm{pH} 7.4)$, dehydrated and embedded in Paraplast X-tra (Sherwood Medical, Deland, FL), and cut to a thickness of $7 \mu \mathrm{m}$ on a rotary microtome (Leica 1512, Wetzlar, Germany).

\section{Regulatory Region Analysis}

The Xenopus tropicalis col2a1 promoter and first intron sequence are from scaffold 161 of the Joint Genome Institute $X$. tropicalis draft genome assembly version 4.1 (http://genome.jgi-psf.org/cgi-bin/ runAlignment? $\mathrm{db}=$ Xentr4). The transcription start site was determined by aligning the complete coding sequence of $X$. tropicalis col2a1 (Genbank Accession: BC063191) with the genomic sequence. A Lagan/ Vista analysis (Brundo et al., 2003) aligned the enhancer sequence between the first introns of mouse Col2a1 (NCBI accession NW_001030577.1; base pair positions 19052638-19048835) and $X$. tropicalis col2a1 (JGI scaffold 161; base pair positions 125221121349). The mouse-specific repeat masker was used for the mouse sequence; the "softmask" option was used for the $X$. tropicalis se- 
quence (http://lagan.stanford.edu).

\section{Preparation of Plasmid Constructs}

Regulatory sequences and egfp were inserted into the pDHG_I-Scel plasmid (Daniel Buchholz, unpublished). This plasmid also contains a gamma-crystallin promoter that drives $g f p$ within the I-Scel recognition sites (Fig. 2D-E). This strong promoter is used to screen injected animals that are successfully transformed with an injected construct (Pan et al., 2006). Egfp was taken from the pCS2+egfp-Bgl2 plasmid (Turner and Weintraub, 1994). The 5'-promoter region and first intron of the Xenopus tropicalis col2a1 gene were amplified by PCR. The forward primer $5^{\prime}-$ GCA CTG CAC ATG TTC TTG CT-3' and reverse primer 5'-GCA GAA TCT TTG TGG GGA AA-3' were used to amplify $\sim 3 \mathrm{~Kb}$ of the $5^{\prime}$ promoter region. The forward primer $5^{\prime}$-GTT CGC AGC CAC ACA AGT C-3' and reverse primer 5'-CCC AGT GTC ACA GAC ACA GAT T-3' were used to amplify $\sim 3.2 \mathrm{~Kb}$ of the first intron. Internal primers were used to amplify 491 bp of the promoter with additional 5 '-Hind III and $3^{\prime}$-Sac1 sites. Intronspecific internal primers were used to amplify four regions of the intron with additional $5^{\prime}$-Sacl and $3^{\prime}$-HindIII sites. The resulting intronic fragments are $347 \mathrm{bp}(\mathrm{A}), 1,517 \mathrm{bp}(\mathrm{B}), 665 \mathrm{bp}(\mathrm{C})$, and $153 \mathrm{bp}$ (D) (Fig. 2B). Whereas regions $B-D$ each overlapped the sequence aligned by Vista analysis, region A did not; it was used as a negative control for enhancer activity. Orientation of each promoter-intron was determined through PCR and sequencing. The four reporter constructs were used for I-Scel meganuclease-mediated transgenics in Xenopus laevis zygotes.

\section{I-Scel Meganuclease-Mediated Transgenics}

The I-Scel transgenic protocol followed those described previously for Xenopus (Ogino et al., 2006 a,b; Pan et al., 2006). A total of $200 \mathrm{ng}$ plasmid DNA was digested for $40 \mathrm{~min}$ at $37^{\circ} \mathrm{C}$ in a $23-\mu \mathrm{L}$ I-Scel meganuclease reaction (New England Biolabs, Ipswich, MA). During this digest fertilized Xenopus laevis eggs were obtained and dejellied using established protocols (Sive et al., 2000). A total of $4.5 \mathrm{~nL}$ of the reaction mix was injected into each single-celled zygote (40 pg DNA/ embryo) using a Femtojet microinjector (Eppendorf, New York). Embryos were raised for $2 \mathrm{hr}$ at room temperature. Individuals that failed to undergo normal cell division were culled. Remaining embryos were raised to embryonic stages 36-40 (Nieuwkoop and Faber, 1994) and screened for GFP expression in the lens under the control of the gamma-crystallin promoter. Incorporation of the transgene after the first cell division yields "half-transgenic" embryos that exhibit unilateral fluorescence on either left or right sides. Incorporation of the transgene before the first cell division yields "full-transgenic" individuals with bilateral transgene expression. Transgenic embryos were raised to early tadpole stages 42-47 and screened for tissue-specific EGFP expression. Tadpoles with intense EGFP expression were raised through metamorphosis to examine reporter expression in adult cartilages.

\section{Antibody Staining and Histology}

Antibody staining was carried out on sectioned material following established Xenopus protocols (Gross et al., 2006). Briefly, tadpoles were fixed overnight in $4 \%$ PFA at $4^{\circ} \mathrm{C}$, embedded in OCT media (Sakura, Torrance, CA), frozen, and sectioned at $10 \mu \mathrm{m}$ on a Leica CM $3050 \mathrm{~S}$ cryostat. Primary antibodies included a FITC-conjugated goat polyclonal antibody to GFP (1:2000; Abcam, Cambridge, MA) and an anti-collagen II monoclonal antibody (1:100; Linsenmeyer and Hendrix, 1980). Slides were counterstained with a $2 \mu \mathrm{g}$ per $\mathrm{mL}$ DAPI nuclear stain solution (Invitrogen) before being cover-slipped with Fluoromount-G (Fisher Scientific, Waltham, MA). Serial sections of post-metamorphic tissues were stained with the Hall-Brunt-Quadruple stain, which colors cartilage blue (Hall, 1986).

\section{Acknowledgements}

Special thanks are due to Daniel Buchholz, who introduced RK to the I-Scel meganuclease-mediated transgenics. Anne Everly helped with microscopy and animal care. Jean-Pierre Saint-Jeannet and Edward De Robertis generously donated the Xenopus laevis sox 9 and col2a1 clones, respectively. Financial support was provided by a Goelet Summer Research Grant from the Museum of Comparative Zoology (RK), NSF grant EF-0334846 (AmphibiaTree; JH), and NSERC grant A5056 (BKH).

\section{References}

AKIYAMA H, CHABOISSIER MC, MARTIN JF, SCHEDL A, DE CROMBRUGGHE B. 2002. The transcription factor Sox9 has essential roles in successive steps of the chondrocyte differentiation pathway and is required for expression of Sox5 and Sox6. Genes Dev 16:2813-2828.

BALTZINGER M, ORI M, PASQUALETTI M, NARDI I, RIJLI FM. 2005. Hoxa2 knockdown in Xenopus results in hyoid to mandibular homeosis. Dev Dyn 234:858-867.

BELL DM, LEUNG KK, WHEATLEY SC, NG LJ, ZHOU S, LING KW, SHAM MH, KOOPMAN P, TAM PP, CHEAH KS. 1997. SOX9 directly regulates the type-II collagen gene. Nat Genet 16:174-178.

BERNASCONI AF. 1951. Über den Ossifikationsmodus bei Xenopus laevis (Daudain). Mem Soc Helvet Sci Nat 79:191-252.

BIEKER JJ, YAZDANI-BUICKY M. 1992. Distribution of type II collagen mRNA in Xenopus embryos visualized by whole-mount in situ hybridization. J Histochem Cytochem 40:1117-1120.

BLITZ IL, ANDELFINGER G, HORB ME. 2005. Germ layers to organs: Using Xenopus to study «later» development. Semin Cell Dev Biol 17:133-145.

BOOT-HANDFORD RP, TUCKWELL DS. 2003. Fibrillar collagen: the key to vertebrate evolution? A tale of molecular incest. Bioessays 25:142-151.

BRIDGEWATER LC, WALKER MD, MILLER GC, ELLISON TA, HOLSINGER LD, POTTER JL, JACKSON TL, CHEN RK, WINKEL VL, ZHANG Z, MCKINNEY S, DE CROMBRUGGHE B. 2003. Adjacent DNA sequences modulate Sox 9 transcriptional activation at paired Sox sites in three chondrocyte-specific enhancer elements. Nuc Acid Res 31:1541-1553.

BRUNDO M, DO C, COOPER G, KIM M, DAVYDOV E, GREEN E, SIDOW A, BATZOGLOU S. 2003. LAGAN and Multi-LAGAN: efficient tools for large-scale multiple alignment of genomic DNA. Genome Res 13:721-731.

CHEN M, LICHTLER AC, SHEU TJ, XIE C, ZHANG X, O'KEEFE RJ, CHEN D. 2007. Generation of a transgenic mouse model with chondrocyte-specific and tamoxifen-inducible expression of Cre recombinase. Genesis 45:44-50.

GOLDRING MB, TSUCHIMOCHI K, IJIRI K. 2006. The control of chondrogenesis $J$ Cell Biochem 97:33-44.

GROSS JB, HANKEN J, OGLESBY E, MARSH-ARMSTRONG N. 2006. Use of a ROSA26:GFP transgenic line for long-term Xenopus fate-mapping studies. $J$ Anat 209:401-413.

GROSS JB, HANKEN J. 2008. Segmentation of the vertebrate skull: neural-crest derivation of adult cartilages in the clawed frog, Xenopus laevis. Int Comp Biol. 48:681-696.

GROVER J, ROUGHLEY PJ. 2006. Generation of a transgenic mouse in which Cre recombinase is expressed under control of the type II collagen promoter and doxycycline administration. Matrix Biol 25:158-165.

HALL BK. 1986. The role of movement and tissue interactions in the development and growth of bone and secondary cartilage in the clavicle of the embryonic chick. J Embryol Exp Morphol 93:133-152.

HALL BK. 2005. Bones and Cartilage: Developmental and Evolutionary Skeletal Biology. Elsevier Academic Press, New York.

HANKEN J. 1992. Life history and morphological evolution. J Evol Bio/ 5:549-557.

HEINEMEYER T, WINGENDER E, REUTER, I, HERMJAKOB H, KEL AE, KEL OV, IGNATIEVA EV, ANANKO EA, PODKOLODNAYA OA, KOLPAKOV FA, PODKOLODNY NL, KOLCHANOV NA 1998. Databases on transcriptional regulation: TRANSFAC, TRRD and COMPEL. Nuc Acid Res 26:362-367.

HORTON W, MIYASHITA T, KOHNO K, HASSELL JR, YAMADA Y. 1987. Identification of a phenotype-specific enhancer in the first intron of the rat collagen II gene. Proc Natl Acad Sci USA 84:8864-8868.

KARSENTY G. 2003. The complexities of skeletal biology. Nature 423:316-318.

KERNEY R, GROSS JB, HANKENJ. 2007. Runx2 is essential for larval hyobranchial cartilage formation in Xenopus laevis. Dev Dyn 236:1650-1662. 
KHOKHA MK, CHUNG C, BUSTAMANTE EL, GAW LW, TROTT KA, YEH J, LIM N, LIN JC, TAVERNER N, AMAYA E, PAPALOPULU N, SMITH JC, ZORN AM, HARLAND RM, GRAMMER TC. 2002. Techniques and probes for the study of Xenopus tropicalis development. Dev Dyn 225:499-510.

KOSHER RA, GAY SW, KAMANITZ JR, KULYK WM, RODGERS BJ, SAI S, TANAKA T, TANZER ML. 1986a. Cartilage proteoglycan core protein gene expression during limb cartilage differentiation. Dev Biol 118:112-117.

KOSHER RA, KULYK WM, GAY SW. 1986b. Collagen gene expression during limb cartilage differentiation. J Cell Biol 102:1151-1156.

LARRAIN J, BACHILLER D, LU B, AGIUS E, PICCOLO S, DE ROBERTIS EM. 2000. BMP-binding modules in chordin: a model for signaling regulation in the extracellular space. Development 127:821-830.

LEFEBVRE $V$, ZHOU G, MUKHOPADHYAY K, SMITH CN, ZHANG Z, EBERSPAECHER H, ZHOU X, SINHA S, MAITY SN, DE CROMBRUGGHE B. 1996. An 18-base-pair sequence in the mouse proalpha1(II) collagen gene is sufficient for expression in cartilage and binds nuclear proteins that are selectively expressed in chondrocytes. Mol Cell Biol 16:4512-4523.

LEFEBVRE V, HUANG W, HARLEY VR, GOODFELLOW PN, DE CROMBRUGGHE B. 1997. SOX9 is a potent activator of the chondrocyte-specific enhancer of the pro alpha1(II) collagen gene. Mol Cell Biol 17:2336-2346.

LEFEBVRE V, SMITS P. 2005. Transcriptional control of chondrocyte fate and differentiation. Birth Defects Res C Embryo Today 75:200-212.

LEUNG KH, NG LJ, HO KY, TAM PPL, CHEAH KE. 1998. Different cis-regulatory DNA elements mediate developmental stage- and tissue-specific expression of the human COL2A1 gene in transgenic mice. J Cell Biol 141:1291-1300.

LINSENMEYER TF, HENDRIX MJ. 1980. Monoclonal antibodies to connective tissue macromolecules: type II collagen. Biochem Biophys Res Commun 92:440-446.

MORIISHIT, SHIBATA Y, TSUKAZAKIT, YAMAGUCHI A. 2005. Expression profile of Xenopus banded hedgehog, a homolog of mouse Indian hedgehog, is related to the late development of endochondral ossification in Xenopus laevis. Biochem Biophys Res Commun 328:867-873.

NAKAMURA E, NGUYEN MT, MACKEM S. 2006. Kinetics of tamoxifen-regulated Cre activity in mice using a cartilage-specific CreER(T) to assay temporal activity windows along the proximodistal limb skeleton. Dev Dyn 235:26032612.

NAKATA K, ONO K, MIYAZAKI J, OLSEN BR, MURAGAKI Y, ADACHI E, YAMAMURA K, KIMURA T. 1993. Osteoarthritis associated with mild chondrodysplasia in transgenic mice expressing alpha 1 (IX) collagen chains with a central deletion. Proc Natl Acad Sci USA 90:2870-2874.

NIEUWKOOP PD, FABER J. 1994. Normal table of Xenopus laevis (Daudin). Garland Publishing, New York.

NUTT SL, BRONCHAIN OJ, HARTLEY KO, AMAYA E. 2001. Comparison of morpholino based translational inhibition during the development of Xenopus laevis and Xenopus tropicalis. Genesis 30:110-113.

OGINOH, MCCONNELL WB, GRAINGER RM. 2006a. High-throughput transgenesis in Xenopus using I-Scel meganuclease. Nat Protoc 1:1703-1710.

OGINO H, MCCONNELL WB, GRAINGER RM. 2006b. Highly efficient transgenesis in Xenopus tropicalis using I-Scel meganuclease. Mech Dev 123:103-113.

OLSSON L, FALCK P, LOPEZ K, COBB J, HANKEN J. 2001. Cranial neural crest cells contribute to connective tissue in cranial muscles in the anuran amphibian, Bombina orientalis. Dev Biol 237:354-367.

OLSSON L, HANKEN J. 1996. Cranial neural-crest migration and chondrogenic fate in the Oriental fire-bellied toad Bombina orientalis: defining the ancestral pattern of head development in anuran amphibians. J Morphol 1996:105-120.

OSAKI M, TAN L, CHOY BK, YOSHIDA Y, CHEAH KS, AURON PE, GOLDRING MB (2003) The TATA-containing core promoter of the type II collagen gene (Col2a1) is the target of interferon- $\gamma$-mediated inhibition in human chondrocytes: requirement for Stat1 $\alpha$, Jak1 and Jak2. Biochem J 369:103-115.

PAN FC, CHEN Y, LOEBER J, HENNINGFELD K, PIELER T. 2006. I-Scel meganuclease-mediated transgenesis in Xenopus. Dev Dyn 235:247-252.

PASK AJ, BEHRINGER RR, RENFREE MB. 2008. Resurrection of DNA function in vivo from an extinct genome. PLOS ONE 3:e2240

PASQUALETTI M, ORI M, NARDI I, RIJLI FM. 2000. Ectopic Hoxa2 induction after neural crest migration results in homeosis of jaw elements in Xenopus. Development 127:5367-5378.
RANKIN SA, HASEBE T, ZORN AM, BUCHHOLZ DR. (2009). Improved Cre reporter transgenic Xenopus. Dev Dyn 238: 2401-2408.

SADAGHIANI B, THIÉBAUD CH. 1987. Neural crest development in the Xenopus laevis embryo, studied by interspecific transplantation and scanning electron microscopy. Dev Biol 124:91-110.

SANDELL LJ, MORRIS N, ROBBINS JR, GOLDRING MB. 1991. Alternatively spliced type II procollagen mRNA's define distinct populations of cells during vertebral development: differential expression of the amino-propeptide. J Cell Biol 114:1307-1319.

SAVAGNER P, MIYASHITA T, YAMADA Y. 1990. Two silencers regulate the tissue-specific expression of the collagen Il gene. J Biol Chem 265:6669-6674.

SAVAGNER P, KREBSBACH PH, HATANO O, MIYASHITA T, LIEBMAN J, YAMADA Y. 1995. Collagen II promoter and enhancer interact synergistically through Sp1 and distinct nuclear factors. DNA Cell Biol 14:501-510.

SEDRA S, MICHAEL M. 1957. The development of the skull, visceral arches, larynx and visceral muscles of the South African clawed toad, Xenopus laevis (Daudin) during the process of metamorphosis (from stage 55 to stage 66). Verh Kon Nederlandse Akad afg Natuurkunde Ser 2:1-80.

SEKIK, FUJIMORIT, SAVAGNER P, HATA A, AIKAWA T, OGATAN, NABESHIMA $Y, K A E C H O O N G$ L. 2003. Mouse snail family transcription repressors regulate chondrocyte, extracellular matrix, type II collagen, and aggrecan. J Biol Chem 278:41862-41870.

SEKIDO R, MURAI K, FUNAHASHI J, KAMACHI Y, FUJISAWA-SEHARA A NABESHIMA Y, KONDOH H. 1994. The delta-crystallin enhancer binding protein delta EF1is a repressor of E2-box-mediated gene activation. Mol Cell Biol 14:5692-5700.

SIVE HL, GRAINGER RM, HARLAND RM. 2000. Early Development of Xenopus laevis. Cold Spring Harbor Laboratory Press, Cold Spring Harbor, New York.

SOBKOW L, EPPERLEIN HH, HERKLOTZ S, STRAUBE WL, TANAKA EM. 2006. A germline GFP transgenic axolotl and its use to track cell fate: dual origin of the fin mesenchyme during development and the fate of blood cells during regeneration. Dev Biol 290:386-397.

SOKOL OM. 1977. The free swimming Pipa larvae with a review of pipid phylogeny (Anura: Pipidae). J Morphol 154:357-426.

SPOKONY RF, AOKIY, SAINT-GERMAIN N, MAGNER-FINKE, SAINT-JEANNET JP. 2002. The transcription factor Sox9 is required for cranial neural crest development in Xenopus. Development 129:421-432.

STONE LS. 1929. Experiments showing the role of migrating neural crest (mesectoderm) in the formation of head skeleton and loose connective tissue in Rana palustris. Wilhelm Roux Arch Entw Mech Org 118:40-77.

STRICKER S, FUNDELE R, VORTKAMP A, MUNDLOS S. 2002. Role of Runx genes in chondrocyte differentiation. Dev Biol 245:95-108.

SU MW, SUZUKI HR, BIEKER JJ, SOLURSH M, RAMIREZ F. 1991. Expression of two nonallelic type II procollagen genes during Xenopus laevis embryogenesis is characterized by stage-specific production of alternatively spliced transcripts. J Cell Biol 115:565-575.

TAKEDA S, BONNAMY JP, OWEN MJ, DUCY P, KARSENTY G. 2001. Continuous expression of Cbfa1 in nonhypertrophic chondrocytes uncovers its ability to induce hypertrophic chondrocyte differentiation and partially rescues Cbfa1deficient mice. Genes Dev 15:467-481.

THERMES V, GRABHER C, RISTORATORE F, BOURRAT F, CHOULIKA A, WITTBRODT J, JOLY JS. 2002. I-Scel meganuclease mediates highly efficient transgenesis in fish. Mech Dev 118:91-98.

THOMSEN GH. 2006. A new century of amphibian developmental biology. Semin Cell Dev Biol 17:78-79.

THOROGOOD P, BEE J, VON DER MARK K. 1986. Transient expression of collagen type II at epitheliomesenchymal interfaces during morphogenesis of the cartilaginous neurocranium. Dev Biol 116:497-509.

TRUEB L, HANKEN J. 1992. Skeletal development in Xenopus laevis (Anura: Pipidae). J Morphol 214:1-41.

TSUMAKI N, TANAKA K, ARIKAWA-HIRASAWA E, NAKASE T, KIMURA T, THOMAS JT, OCHI T, LUYTEN FP, YAMADA Y. 1999. Role of CDMP-1 in skeletal morphogenesis: promotion of mesenchymal cell recruitment and chondrocyte differentiation. J Cell Biol 144:161-173.

TURNER DL, WEINTRAUB H. 1994. Expression of achaete-scute homolog 3 in Xenopus embryos converts ectodermal cells to a neural fate. Genes Dev 
8:1434-1447

UETA C, IWAMOTO M, KANATANI N, YOSHIDA C, LIU Y, ENOMOTO-IWAMOTO $M$, OHMORI T, ENOMOTO H, NAKATA K, TAKADA K, KURISU K, KOMORI T. 2001. Skeletal malformations caused by overexpression of Cbfa1 or its dominant negative form in chondrocytes. J Cell Biol 153:87-100.

WOOD A, ASHHURST DE, CORBETT A, THOROGOOD P. 1991. The transient expression of type II collagen at tissue interfaces during mammalian craniofacial development. Development 111:955-968.

ZELZER E, GLOTZER DJ, HARTMAN C, THOMAS D, FUKAI N, SOKER S, OLSEN B. 2001. Tissue specific regulation of VEGF expression during bone development requires CBFa-1/RUNX2. Mech Dev 106:97-106.
ZELZER E, OLSEN B. 2003. Genetic basis of skeletal diseases. Nature 423:343348.

ZHOU G, GAROFALO S, MUKHOPADHYAY K, LEFEBVRE V, SMITH CN, EBERSPAECHER H, DE CROMBRUGGHE B. 1995. A $182 \mathrm{bp}$ fragment of the mouse pro alpha 1 (II) collagen gene is sufficient to direct chondrocyte expression in transgenic mice. J Cell Sci 108:3677-3684.

ZHOU G, LEFEBVRE V, ZHANG Z, EBERSPAECHER H, DE CROMBRUGGHE B 1998. Three high mobility group-like sequences within a 48-base pair enhancer of the Col2a1 gene are required for cartilage-specific expression in vivo. J Biol Chem 273:14989-14997.

\section{Further Related Reading, published previously in the Int. J. Dev. Biol.}

See Special Issue Pattern Formation edited by Michael K. Richardson and Cheng-Ming Chuong at: http://www.ijdb.ehu.es/web/contents.php?vol=53\&issue=5-6

Loss of Sox9 function results in defective chondrocyte differentiation of mouse embryonic stem cells in vitro

Gunnar Hargus, Ralf Kist, Jan Kramer, Daniela Gerstel, Angela Neitz, Gerd Scherer and Jürgen Rohwedel

Int. J. Dev. Biol. (2008) 52: 323-332

BMP4 promotes chondrocyte proliferation and hypertrophy in the endochondral cranial base.

Lillian Shum, Xibin Wang, Alex A Kane and Glen H Nuckolls

Int. J. Dev. Biol. (2003) 47: 423-431

Analysis of the odontogenic and osteogenic potentials of dental pulp in vivo using a Col1a1-2.3-GFP transgene.

Alen Braut, Edward J Kollar and Mina Mina

Int. J. Dev. Biol. (2003) 47: 281-29

Spatiotemporal pattern of the mouse chondromodulin-I gene expression and its regulatory role in vascular invasion into cartilage during endochondral bone formation. C Shukunami, K lyama, $\mathrm{H}$ Inoue and $\mathrm{Y}$ Hiraki

Int. J. Dev. Biol. (1999) 43: 39-49

Sequential synthesis of cartilage and bone marker proteins during transdifferentiation of mouse Meckel's cartilage chondrocytes in vitro.

K Ishizeki, Y Hiraki, M Kubo and T Nawa

Int. J. Dev. Biol. (1997) 41: 83-89

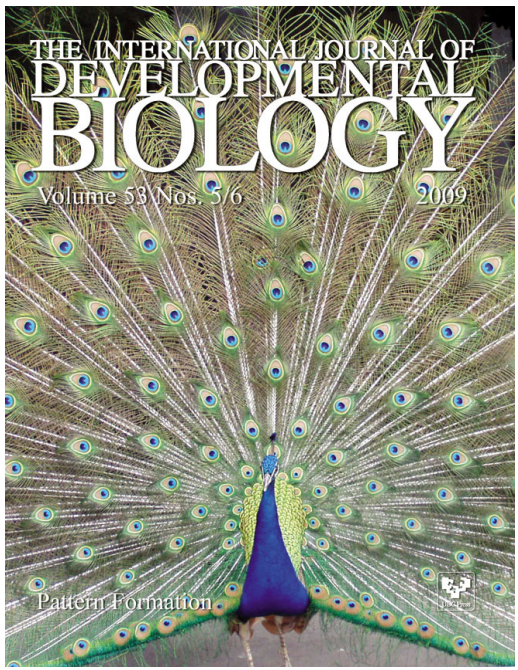

5 yr ISI Impact Factor $(2008)=3.271$

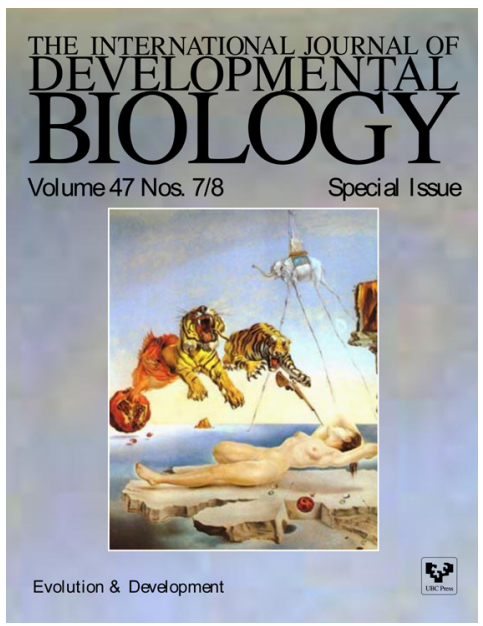

